\title{
ABSORBING ELEMENTS IN LATTICE MODULES
}

\author{
C. S. Manjarekar and A. V. Bingi \\ Received: 21 April 2015; Revised: 8 December 2015 \\ Communicated by Abdullah Harmancı
}

\begin{abstract}
In this paper we introduce and investigate 2-absorbing, $n$-absorbing, $(n, k)$-absorbing, weakly 2 -absorbing, weakly $n$-absorbing and weakly $(n, k)$ absorbing elements in a lattice module $M$. Some characterizations of 2absorbing and weakly 2-absorbing elements of $M$ are obtained. By counter example it is shown that a weakly 2-absorbing element of $M$ need not be 2absorbing. Finally we show that if $N \in M$ is a 2-absorbing element, then $\operatorname{rad}(N)$ is a 2-absorbing element of $M$.
\end{abstract}

Mathematics Subject Classification (2010): 06D10, 06E10, 06E99, 06F99 Keywords: 2-absorbing element, $n$-absorbing element, $(n, k)$-absorbing element, weakly 2-absorbing element, $\operatorname{rad}(N)$

\section{Introduction}

The concept of 2-absorbing and weakly 2-absorbing ideals in commutative rings was introduced by A. Badawi in [4] and A. Badawi et. al. in [5] respectively as a generalization of prime and weakly prime ideals. D. F. Anderson et. al. in [3] generalized the concept of 2-absorbing ideals to $n$-absorbing ideals. A. Y. Darani et. al. in [8] generalized the concept of 2-absorbing and weakly 2-absorbing ideals to submodules of a module over a commutative ring. Further this concept was extended to $n$-absorbing submodules by A. Y. Darani et. al. in [9]. In multiplicative lattices, the study of 2-absorbing elements and weakly 2-absorbing elements was done by C. Jayaram et. al. in [11] while the study of $n$-absorbing elements and weakly $n$-absorbing elements was done by S. Ballal et. al. in [6]. Our aim is to extend the notion of absorbing elements in a multiplicative lattice to a notion of absorbing elements in lattice modules and study its properties.

A multiplicative lattice $L$ is a complete lattice provided with commutative, associative and join distributive multiplication in which the largest element 1 acts as a multiplicative identity. An element $e \in L$ is called meet principal if $a \wedge b e=$ $((a: e) \wedge b) e$ for all $a, b \in L$. An element $e \in L$ is called join principal if $(a e \vee b): e=(b: e) \vee a$ for all $a, b \in L$. An element $e \in L$ is called principal 
if $e$ is both meet principal and join principal. An element $a \in L$ is called compact if for $X \subseteq L, a \leqslant \vee X$ implies the existence of a finite number of elements $a_{1}, a_{2}, \cdots, a_{n}$ in $X$ such that $a \leqslant a_{1} \vee a_{2} \vee \cdots \vee a_{n}$. The set of compact elements of $L$ will be denoted by $L_{*}$. If each element of $L$ is a join of compact elements of $L$, then $L$ is called a compactly generated lattice or simply a $C G$-lattice. $L$ is said to be a principally generated lattice or simply a $P G$-lattice if each element of $L$ is the join of principal elements of $L$. Throughout this paper $L$ denotes a compactly generated multiplicative lattice with 1 compact in which every finite product of compact elements is compact.

An element $a \in L$ is said to be proper if $a<1$. A proper element $p \in L$ is called a prime element if $a b \leqslant p$ implies $a \leqslant p$ or $b \leqslant p$ where $a, b \in L$ and is called $a$ primary element if $a b \leqslant p$ implies $a \leqslant p$ or $b^{n} \leqslant p$ for some $n \in \mathbb{Z}_{+}$where $a, b \in L_{*}$. A proper element $p \in L$ is called a weakly prime element if $0 \neq a b \leqslant p$ implies $a \leqslant p$ or $b \leqslant p$ where $a, b \in L$ and is called a weakly primary element if $0 \neq a b \leqslant p$ implies $a \leqslant p$ or $b^{n} \leqslant p$ for some $n \in \mathbb{Z}_{+}$where $a, b \in L_{*}$. A proper element $q \in L$ is called p-primary if $q$ is primary and $p=\sqrt{q}$ is prime. A proper element $q \in L$ is called $p$-weakly primary if $q$ is weakly primary and $p=\sqrt{q}$ is weakly prime. For $a, b \in L,(a: b)=\vee\{x \in L \mid x b \leqslant a\}$. The radical of $a \in L$ is denoted by $\sqrt{a}$ and is defined as $\vee\left\{x \in L_{*} \mid x^{n} \leqslant a\right.$, for some $\left.n \in \mathbb{Z}_{+}\right\}$. An element $a \in L$ is said to be nilpotent if $a^{n}=0$ for some $n \in \mathbb{Z}_{+}$. A multiplicative lattice $L$ is said to be $a$ reduced lattice if $0 \in L$ is the only nilpotent element of $L$. The reader is referred to [2] for general background and terminology in multiplicative lattices.

Let $M$ be a complete lattice and $L$ be a multiplicative lattice. Then $M$ is called $L$-module or module over $L$ if there is a multiplication between elements of $L$ and $M$ written as $a B$ where $a \in L$ and $B \in M$ which satisfies the following properties: (1) $\left.\underset{\alpha}{\vee} a_{\alpha}\right) A=\underset{\alpha}{\vee} a_{\alpha} A,(2) a\left(\underset{\alpha}{\vee} A_{\alpha}\right)=\underset{\alpha}{\vee} a A_{\alpha}$, (3) $(a b) A=a(b A)$, (4) $1 A=A$, (5)

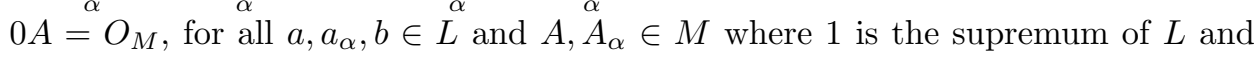
0 is the infimum of $L$. We denote by $O_{M}$ and $I_{M}$ for the least element and the greatest element of $M$, respectively. Elements of $L$ will generally be denoted by $a, b, c, \cdots$ and elements of $M$ will generally be denoted by $A, B, C, \cdots$.

Let $M$ be an $L$-module. For $N \in M$ and $a \in L,(N: a)=\vee\{X \in M \mid a X \leqslant$ $N\}$. For $A, B \in M,(A: B)=\vee\{x \in L \mid x B \leqslant A\}$. An $L$-module $M$ is called a multiplication lattice module if for every element $N \in M$ there exists an element $a \in L$ such that $N=a I_{M}$. An element $N \in M$ is said to be proper if $N<I_{M}$. A proper element $N \in M$ is said to be prime if for $a \in L$ and $X \in M ; a X \leqslant N$ implies $X \leqslant N$ or $a \leqslant\left(N: I_{M}\right)$. A proper element $N \in M$ is said to be weakly prime if for $a \in L$ and $X \in M ; O_{M} \neq a X \leqslant N$ implies $X \leqslant N$ or $a \leqslant\left(N: I_{M}\right)$. 
If $N \in M$ is a prime element, then $\left(N: I_{M}\right)$ is a prime element in $L$. An element $N<I_{M}$ in $M$ is said to be primary if for $a \in L$ and $X \in M ; a X \leqslant N$ implies $X \leqslant N$ or $a^{n} \leqslant\left(N: I_{M}\right)$ for some $n \in \mathbb{Z}_{+}$. An element $N<I_{M}$ in $M$ is said to be weakly primary if for $a \in L$ and $X \in M ; O_{M} \neq a X \leqslant N$ implies $X \leqslant N$ or $a^{n} \leqslant\left(N: I_{M}\right)$ for some $n \in \mathbb{Z}_{+}$. A proper element $N \in M$ is said to be $p$-primary if $N$ is primary and $p=\sqrt{N: I_{M}}$ is prime. A proper element $N \in M$ is said to be $p$-weakly primary if $N$ is weakly primary and $p=\sqrt{N: I_{M}}$ is weakly prime. An element $N \in M$ is called a radical element if $\left(N: I_{M}\right)=\sqrt{\left(N: I_{M}\right)}$ where $\sqrt{\left(N: I_{M}\right)}=\vee\left\{x \in L_{*} \mid x^{n} \leqslant\left(N: I_{M}\right)\right.$, for some $\left.n \in \mathbb{Z}_{+}\right\}=\vee\left\{x \in L_{*} \mid\right.$ $x^{n} I_{M} \leqslant N$, for some $\left.n \in \mathbb{Z}_{+}\right\}$. An element $N \in M$ is called meet principal if $(b \wedge(B: N)) N=b N \wedge B$ for all $b \in L, B \in M$. An element $N \in M$ is called join principal if $b \vee(B: N)=((b N \vee B): N)$ for all $b \in L, B \in M$. An element $N \in M$ is said to be principal if $N$ is both meet principal and join principal. An element $N \in M$ is called compact if $N \leqslant \vee_{\alpha} A_{\alpha}$ implies $N \leqslant A_{\alpha_{1}} \vee A_{\alpha_{2}} \vee \cdots \vee A_{\alpha_{n}}$ for some finite subset $\left\{\alpha_{1}, \alpha_{2}, \cdots, \alpha_{n}\right\}$. The set of compact elements of $M$ is denoted by $M_{*}$. If each element of $M$ is a join of compact elements of $M$, then $M$ is called a $C G$ lattice $L$-module. If $\left(O_{M}: I_{M}\right)=0$, then $M$ is called a faithful $L$-module. $M$ is said to be a $P G$-lattice $L$-module if each element of $M$ is the join of principal elements of $M$. For all the definitions in a lattice module and some other definitions, one can refer [7].

According to [11], a proper element $q \in L$ is said to be a 2-absorbing element if for every $a, b, c \in L ; a b c \leqslant q$ implies either $a b \leqslant q$ or $b c \leqslant q$ or $c a \leqslant q$ and a proper element $q \in L$ is said to be a weakly 2-absorbing element if for every $a, b, c \in L ; 0 \neq a b c \leqslant q$ implies either $a b \leqslant q$ or $b c \leqslant q$ or $c a \leqslant q$. Obviously a prime element of $L$ is a 1-absorbing element and a weakly prime element of $L$ is a weakly 1-absorbing element. According to [6], a proper element of $q \in L$ is said to be a $n$-absorbing element if for every $a_{1}, a_{2}, \cdots, a_{n}, a_{n+1} \in L ; a_{1} a_{2} \cdots a_{n} a_{n+1} \leqslant q$ implies there are $n$ of $a_{i}^{\prime} s$ whose product is less than or equal to $q$, that is, $\widehat{a}_{i} \leqslant q$ for some $i(1 \leqslant i \leqslant(n+1))$ where $\widehat{a}_{i}$ is the element $a_{1} \cdots a_{i-1} a_{i+1} \cdots a_{n} a_{n+1}$ and a proper element of $q \in L$ is said to be a weakly $n$-absorbing element if for every $a_{1}, a_{2}, \cdots, a_{n}, a_{n+1} \in L ; 0 \neq a_{1} a_{2} \cdots a_{n} a_{n+1} \leqslant q$ implies there are $n$ of $a_{i}^{\prime} s$ whose product is less than or equal to $q$. In this paper we introduce and investigate 2-absorbing, $n$-absorbing, $(n, k)$-absorbing, weakly 2 -absorbing, weakly $n$-absorbing and weakly $(n, k)$-absorbing elements in a lattice module $M$. We give characterization for 2-absorbing and weakly 2-absorbing elements of $M$. By counter example we show that a weakly 2 -absorbing element of $M$ need not be 2 -absorbing. We establish a condition for a weakly 2 -absorbing element of $M$ to be a 2 -absorbing 
element. Finally we show if $N \in M$ is a 2-absorbing element then $\operatorname{rad}(N)$ is a 2absorbing element of $M$.

This paper is motivated by [8] and [9]. Many of the results obtained in this paper are versions of results in [8] and [9]. It should be mentioned that there is a significant difference between our results and the already existing ones presented in [8] and [9], as principal elements of $M$ are used in these proofs. We have generalized the important results of a multiplication module over a commutative ring obtained in [10] to a multiplication lattice module $M$ over a multiplicative lattice $L$, using the principal elements so as to establish the results of $\operatorname{rad}(N)$.

\section{Absorbing elements in $M$}

In this section we introduce and study absorbing elements of an $L$-module $M$. We begin with the following definitions.

Definition 2.1. A proper element $N$ of an $L$-module $M$ is said to be 2-absorbing if for every $a, b \in L$ and $Q \in M ; a b Q \leqslant N$ implies either $a b \leqslant\left(N: I_{M}\right)$ or $a Q \leqslant N$ or $b Q \leqslant N$.

Obviously a prime element of an $L$-module $M$ is a 2-absorbing element. Also a prime element of $M$ can be thought of as a 1-absorbing element.

Definition 2.2. A proper element $N$ of an $L$-module $M$ is said to be weakly 2-absorbing if for every $a, b \in L$ and $Q \in M ; O_{M} \neq a b Q \leqslant N$ implies either $a b \leqslant\left(N: I_{M}\right)$ or $a Q \leqslant N$ or $b Q \leqslant N$.

Definition 2.3. Let $n \in \mathbb{Z}_{+}$. A proper element $N$ of an $L$-module $M$ is said to be $n$-absorbing if for every $a_{1}, a_{2}, \cdots, a_{n} \in L$ and $Q \in M ; a_{1} a_{2} \cdots a_{n} Q \leqslant N$ implies either $a_{1} a_{2} \cdots a_{n} \leqslant\left(N: I_{M}\right)$ or there are (n-1) of $a_{i}^{\prime} s$ whose product with $Q$ is less than or equal to $N$, that is, either $a_{1} a_{2} \cdots a_{n} \leqslant\left(N: I_{M}\right)$ or $\widehat{a}_{i} Q \leqslant N$ for some $i(1 \leqslant i \leqslant n)$ where $\widehat{a}_{i}$ is the element $a_{1} \cdots a_{i-1} a_{i+1} \cdots a_{n}$.

Definition 2.4. Let $n \in \mathbb{Z}_{+}$. A proper element $N$ of an $L$-module $M$ is said to be weakly $n$-absorbing if for every $a_{1}, a_{2}, \cdots, a_{n} \in L$ and $Q \in M ; O_{M} \neq a_{1} a_{2} \cdots a_{n} Q \leqslant N$ implies either $a_{1} a_{2} \cdots a_{n} \leqslant\left(N: I_{M}\right)$ or there are (n-1) of $a_{i}^{\prime} s$ whose product with $Q$ is less than or equal to $N$, that is, either $a_{1} a_{2} \cdots a_{n} \leqslant\left(N: I_{M}\right)$ or $\widehat{a}_{i} Q \leqslant N$ for some $i(1 \leqslant i \leqslant n)$ where $\widehat{a}_{i}$ is the element $a_{1} \cdots a_{i-1} a_{i+1} \cdots a_{n}$.

Definition 2.5. Let $n, k \in \mathbb{Z}_{+}$where $n>k$. A proper element $N$ of an $L$ module $M$ is said to be $(n, k)$-absorbing if for every $a_{1}, a_{2}, \cdots, a_{n} \in L$ and $Q \in M$; $a_{1} a_{2} \cdots a_{n} Q \leqslant N$ implies either there are $k$ of the $a_{i}^{\prime} s$ whose product is less than 
or equal to $\left(N: I_{M}\right)$ or there are $(k-1)$ of the $a_{i}^{\prime} s$ whose product with $Q$ is less than or equal to $N$.

Definition 2.6. Let $n, k \in \mathbb{Z}_{+}$where $n>k$. A proper element $N$ of an $L$-module $M$ is said to be weakly $(n, k)$-absorbing if for every $a_{1}, a_{2}, \cdots, a_{n} \in L$ and $Q \in M$; $O_{M} \neq a_{1} a_{2} \cdots a_{n} Q \leqslant N$ implies either there are $k$ of the $a_{i}^{\prime} s$ whose product is less than or equal to $\left(N: I_{M}\right)$ or there are $(k-1)$ of the $a_{i}^{\prime} s$ whose product with $Q$ is less than or equal to $N$.

Now we give the characterization of a 2-absorbing element of $M$.

Theorem 2.7. Let $M$ be a CG-lattice L-module and $N$ be a proper element $M$. Then the following statements are equivalent:

(1) $N$ is a 2-absorbing element of $M$.

(2) for every $a, b \in L$ and $Q \in M$ such that $N \leqslant Q$; abQ $\leqslant N$ implies either $a b \leqslant\left(N: I_{M}\right)$ or $a Q \leqslant N$ or $b Q \leqslant N$.

(3) for every $a, b \in L$ such that $a b \nless\left(N: I_{M}\right)$; either $(N: a b)=(N: a)$ or $(N: a b)=(N: b)$.

(4) for any elements $r, s \in L_{*}, K \in M_{*}$; if $r s K \leqslant N$ then either $r s \leqslant(N$ : $\left.I_{M}\right)$ or $r K \leqslant N$ or $s K \leqslant N$.

Proof. $(1) \Rightarrow(2)$ It is obvious.

$(2) \Rightarrow(3)$ Suppose $(2)$ holds. Let $K \in M$ be such that $K \leqslant(N: a b)$ and $a b \nless$ $\left(N: I_{M}\right)$ for $a, b \in L$. Then $a b K \leqslant N$. Clearly $a b(K \vee N)=(a b K) \vee(a b N) \leqslant N$. Let $U=K \vee N$. Now as $N \leqslant U, a b U \leqslant N$ and $a b \nless\left(N: I_{M}\right)$; by (2) it follows that either $a U \leqslant N$ or $b U \leqslant N$ which implies either $a K \leqslant N$ or $b K \leqslant N$ and so either $K \leqslant(N: a)$ or $K \leqslant(N: b)$. Hence we have either $(N: a b) \leqslant(N: a)$ or $(N: a b) \leqslant(N: b)$. Obviously $(N: a) \leqslant(N: a b)$ and $(N: b) \leqslant(N: a b)$. Thus either $(N: a b)=(N: a)$ or $(N: a b)=(N: b)$.

$(3) \Rightarrow(4)$ Suppose (3) holds. Let $r s K \leqslant N$ and $r s \nless\left(N: I_{M}\right)$ for $r, s \in L_{*}$, $K \in M_{*}$. Then by $(3)$ we have either $(N: r s)=(N: r)$ or $(N: r s)=(N: s)$. So as $K \leqslant(N: r s)$ we have either $K \leqslant(N: r)$ or $K \leqslant(N: s)$. Thus either $r K \leqslant N$ or $s K \leqslant N$.

$(4) \Rightarrow(1)$ Suppose (4) holds. Let $a b X \leqslant N, a X \nless N$ and $b X \nless N$ for $a, b \in$ $L, X \in M$. As $L$ and $M$ are compactly generated, there exist $r, s \in L_{*}$ and $Y, Y^{\prime} \in$ $M_{*}$ such that $r \leqslant a, s \leqslant b, Y \leqslant X, Y^{\prime} \leqslant X, r Y^{\prime} \nless N$ and $s Y^{\prime} \nless N$. Then $r s \leqslant a b$. Now $r, s \in L_{*},\left(Y \vee Y^{\prime}\right) \in M_{*}$ such that $r s\left(Y \vee Y^{\prime}\right) \leqslant a b X \leqslant N, r\left(Y \vee Y^{\prime}\right) \nless N$ and $s\left(Y \vee Y^{\prime}\right) \nless N$. So by (4) rs $\leqslant\left(N: I_{M}\right)$ which implies $a b \leqslant\left(N: I_{M}\right)$. Therefore $N$ is a 2-absorbing element of $M$. 
A similar characterization of a weakly 2-absorbing element of $M$ is as follows.

Theorem 2.8. Let $M$ be a CG-lattice L-module and $N$ be a proper element of $M$. Then the following statements are equivalent:

(1) $N$ is a weakly 2-absorbing element of $M$.

(2) For every $a, b \in L$ and $Q \in M$ such that $N \leqslant Q ; O_{M} \neq a b Q \leqslant N$ implies either $a b \leqslant\left(N: I_{M}\right)$ or $a Q \leqslant N$ or $b Q \leqslant N$.

(3) For every $a, b \in L$ such that $a b \nless\left(N: I_{M}\right)$; either $(N: a b)=\left(O_{M}: a b\right)$ or $(N: a b)=(N: a)$ or $(N: a b)=(N: b)$.

(4) For every $r, s \in L_{*}, K \in M_{*}$; if $O_{M} \neq r s K \leqslant N$ then either $r \leqslant(N$ : $\left.I_{M}\right)$ or $r K \leqslant N$ or $s K \leqslant N$.

Proof. (1) $\Rightarrow(2)$ It is obvious.

$(2) \Rightarrow(3)$ Suppose (2) holds. Let $K \in M$ be such that $K \leqslant(N: a b)$ and $a b \nless\left(N: I_{M}\right)$ for $a, b \in L$. Then $a b K \leqslant N$. If $a b K=O_{M}$, then $K \leqslant\left(O_{M}: a b\right)$. If $a b K \neq O_{M}$, then $O_{M} \neq a b(K \vee N)=(a b K) \vee(a b N) \leqslant N$. Let $U=K \vee N$. Now as $N \leqslant U, O_{M} \neq a b U \leqslant N$ and $a b \nless\left(N: I_{M}\right)$; by (2) it follows that either $a U \leqslant N$ or $b U \leqslant N$ which implies either $a K \leqslant N$ or $b K \leqslant N$ and so either $K \leqslant(N: a)$ or $K \leqslant(N: b)$. Hence we have either $(N: a b) \leqslant\left(O_{M}: a b\right)$ or $(N: a b) \leqslant(N: a)$ or $(N: a b) \leqslant(N: b)$. Obviously $\left(O_{M}: a b\right) \leqslant(N: a b),(N: a) \leqslant(N: a b)$ and $(N: b) \leqslant(N: a b)$. Thus either $(N: a b)=\left(O_{M}: a b\right)$ or $(N: a b)=(N: a)$ or $(N: a b)=(N: b)$.

$(3) \Rightarrow(4)$ Suppose (3) holds. Let $O_{M} \neq r s K \leqslant N$ and $r s \nless\left(N: I_{M}\right)$ for $r, s \in$ $L_{*}, K \in M_{*}$. Then by (3) we have either $(N: r s)=(N: r)$ or $(N: r s)=(N: s)$ or $(N: r s)=\left(O_{M}: r s\right)$. Since $K \leqslant(N: r s)$ it follows that either $K \leqslant\left(O_{M}: r s\right)$ or $K \leqslant(N: r)$ or $K \leqslant(N: s)$. As $K \leqslant\left(O_{M}: r s\right)$ gives $r s K=O_{M}$, a contradiction, we must have either $K \leqslant(N: r)$ or $K \leqslant(N: s)$ which implies either $r K \leqslant N$ or $s K \leqslant N$.

$(4) \Rightarrow(1)$ Suppose (4) holds. Let $O_{M} \neq a b X \leqslant N, a X \nless N$ and $b X \nless N$ for $a, b \in L, X \in M$. As $L$ and $M$ are compactly generated, there exist $r, s \in L_{*}$ and $Y, Y^{\prime} \in M_{*}$ such that $r \leqslant a, s \leqslant b, Y \leqslant X, Y^{\prime} \leqslant X, r Y^{\prime} \nless N, s Y^{\prime} \nless$ $N$ and $O_{M} \neq r s Y^{\prime}$. Then $r s \leqslant a b$. Now $r, s \in L_{*},\left(Y \vee Y^{\prime}\right) \in M_{*}$ such that $O_{M} \neq r s\left(Y \vee Y^{\prime}\right) \leqslant a b X \leqslant N, r\left(Y \vee Y^{\prime}\right) \nless N$ and $s\left(Y \vee Y^{\prime}\right) \nless N$. So by (4) $r s \leqslant\left(N: I_{M}\right)$ which implies $a b \leqslant\left(N: I_{M}\right)$. Therefore $N$ is a weakly 2-absorbing element of $M$.

In the next theorem, we show that the meet and join of a family of ascending chain of 2-absorbing elements of $M$ are again 2-absorbing. 
Theorem 2.9. Let $\left\{N_{i} \mid i \in \mathbb{Z}_{+}\right\}$be a (ascending or descending) chain of 2absorbing elements of an L-module $M$. Then

(1) $\wedge_{i \in \mathbb{Z}_{+}} N_{i}$ is a 2-absorbing element of $M$.

(2) $\underset{i \in \mathbb{Z}_{+}}{\vee} N_{i}$ is a 2-absorbing element of $M$ if $I_{M}$ is compact.

Proof. Let $N_{1} \leqslant N_{2} \leqslant \cdots \leqslant N_{i} \leqslant \cdots$ be an ascending chain of 2-absorbing elements of $M$.

(1) Clearly, $\left(\underset{i \in \mathbb{Z}_{+}}{\wedge} N_{i}\right) \neq I_{M}$. Let $a b Q \leqslant\left(\wedge_{i \in \mathbb{Z}_{+}} N_{i}\right)$ and $a Q \nless\left(\wedge_{i \in \mathbb{Z}_{+}}^{\wedge} N_{i}\right)$ for $a, b \in L$, $Q \in M$. Then $a Q \nless N_{j}$ for some $j \in \mathbb{Z}_{+}$but $a b Q \leqslant N_{j}$ which implies $a b \leqslant\left(N_{j}\right.$ : $\left.I_{M}\right)$ or $b Q \leqslant N_{j}$ as $N_{j}$ is a 2-absorbing element. Now let $N_{i} \neq N_{j}$. Then as $\left\{N_{i}\right\}$ is a chain we have either $N_{i}<N_{j}$ or $N_{j}<N_{i}$. If $N_{i}<N_{j}$ then as $N_{i}$ is a 2-absorbing element, $a b Q \leqslant N_{i}$ and $a Q \nless N_{i}$ we have either $a b \leqslant\left(N_{i}: I_{M}\right)$ or $b Q \leqslant N_{i}$. If $N_{j}<N_{i}$ then either $a b \leqslant\left(N_{j}: I_{M}\right) \leqslant\left(N_{i}: I_{M}\right)$ or $b Q \leqslant N_{j}<N_{i}$. Thus either $a b \leqslant \wedge_{i \in \mathbb{Z}_{+}}\left(N_{i}: I_{M}\right)=\left[\left(\wedge_{i \in \mathbb{Z}_{+}}^{\wedge} N_{i}\right): I_{M}\right]$ or $b Q \leqslant \wedge_{i \in \mathbb{Z}_{+}} N_{i}$ which proves that $\wedge_{i \in \mathbb{Z}_{+}} N_{i}$ is a 2-absorbing element of $M$.

(2) Since $I_{M}$ is compact, $\left(\underset{i \in \mathbb{Z}_{+}}{\vee} N_{i}\right) \neq I_{M}$. Let $a b Q \leqslant\left(\underset{i \in \mathbb{Z}_{+}}{\vee} N_{i}\right)$ and $a Q \nless\left(\underset{i \in \mathbb{Z}_{+}}{\vee} N_{i}\right)$ for $a, b \in L, Q \in M$. Then as $\left\{N_{i}\right\}$ is a chain we have $a b Q \leqslant N_{j}$ for some $j \in \mathbb{Z}_{+}$ but $a Q \nless N_{j}$ which implies either $a b I_{M} \leqslant N_{j} \leqslant\left(\underset{i \in \mathbb{Z}_{+}}{\vee} N_{i}\right)$ or $b Q \leqslant N_{j} \leqslant\left(\underset{i \in \mathbb{Z}_{+}}{\vee} N_{i}\right)$ as $N_{j}$ is a 2-absorbing element and thus $\underset{i \in \mathbb{Z}_{+}}{\vee} N_{i}$ is a 2-absorbing element of $M$.

The "weakly" version of above Theorem 2.9 is as follows.

Theorem 2.10. Let $\left\{N_{i} \mid i \in \mathbb{Z}_{+}\right\}$be a (ascending or descending) chain of weakly 2-absorbing elements of an L-module $M$. Then

(1) $\underset{i \in \mathbb{Z}}{\wedge} N_{i}$ is a weakly 2-absorbing element of $M$.

(2) $\underset{i \in \mathbb{Z}_{+}}{\vee} N_{i}$ is a weakly 2-absorbing element of $M$ if $I_{M}$ is compact.

Proof. The proof is similar to the proof of Theorem 2.9 and hence omitted.

Theorem 2.11. If a proper element $N$ of an $L$-module $M$ is a 2-absorbing element then $(N: d)$ is a 2-absorbing element of $M$ for every $d \in L$.

Proof. Let $d, a, b \in L$ and $Q \in M$. Assume that $a b Q \leqslant(N: d), a Q \nless(N: d)$ and $b Q \nless(N: d)$. As $a b(d Q) \leqslant N, a(d Q) \nless N, b(d Q) \nless N$ and $N \in M$ is 2 absorbing we get $a b I_{M} \leqslant N$ which implies $d\left(a b I_{M}\right) \leqslant N$. It follows that $a b \leqslant\left((N: d): I_{M}\right)$ and hence $(N: d)$ is a 2 -absorbing element of $M$.

The following theorem shows that if an element in $M$ (or $L$ ) is 2-absorbing then its corresponding element in $L$ (or $M$ ) is also 2-absorbing. 
Theorem 2.12. Let $M$ be a faithful multiplication $P G$-lattice L-module with $I_{M}$ compact where $L$ is also a PG-lattice. Then the following statements are equivalent:

(1) $N$ is a 2-absorbing element of $M$.

(2) $\left(N: I_{M}\right)$ is a 2-absorbing element of $L$.

(3) $N=q I_{M}$ for some 2-absorbing element $q \in L$.

Proof. (1) $\Rightarrow(2)$ Assume that $N$ is a 2-absorbing element of $M$. Let $a b c \leqslant$ $\left(N: I_{M}\right)$ such that $a b \nless\left(N: I_{M}\right)$ and $b c \nless\left(N: I_{M}\right)$ for $a, b, c \in L$. Then as $a c\left(b I_{M}\right) \leqslant N, a\left(b I_{M}\right) \nless N, c\left(b I_{M}\right) \nless N$ and $N$ is a 2-absorbing element we have $a c \leqslant\left(N: I_{M}\right)$ which implies $\left(N: I_{M}\right)$ is a 2-absorbing element of $L$.

$(2) \Rightarrow(1)$ Assume that $\left(N: I_{M}\right)$ is a 2-absorbing element of $L$. Let abQ $\leqslant N$ for $a, b \in L, Q \in M$. Since $M$ is a multiplication lattice $L$-module, $Q=q I_{M}$ for some $q \in L$. Then as $a b q \leqslant\left(N: I_{M}\right)$ and $\left(N: I_{M}\right)$ is a 2-absorbing element we have either $a b \leqslant\left(N: I_{M}\right)$ or $b q \leqslant\left(N: I_{M}\right)$ or $a q \leqslant\left(N: I_{M}\right)$ which implies either $a b \leqslant\left(N: I_{M}\right)$ or $b Q \leqslant N$ or $a Q \leqslant N$ and hence $N \in M$ is a 2-absorbing element.

$(2) \Rightarrow(3)$ Assume that $\left(N: I_{M}\right)$ is a 2-absorbing element of $L$. Then obviously (3) holds since in a multiplication lattice $L$-module $M$ we have $N=\left(N: I_{M}\right) I_{M}$.

(3) $\Rightarrow(2)$ Assume that $N=q I_{M}$ for some 2-absorbing element $q \in L$. Also $N=\left(N: I_{M}\right) I_{M}$ since $M$ is a multiplication lattice $L$-module. It follows that $q I_{M}=\left(N: I_{M}\right) I_{M}$. As $I_{M}$ is compact, (2) holds by Theorem 5 of [7].

In view of above Theorem 2.12 we give the following corollary without proof.

Corollary 2.13. If a proper element $N$ of an $L$-module $M$ is 2-absorbing, then $\left(N: I_{M}\right)$ is a 2-absorbing element of $L$. The converse holds if $M$ is a multiplication lattice $L$-module.

The above Corollary 2.13 is true for "weakly" version provided $M$ is faithful as shown below.

Theorem 2.14. If a proper element $N$ of a faithful L-module $M$ is weakly 2absorbing, then $\left(N: I_{M}\right)$ is a weakly 2-absorbing element of $L$. The converse holds if $M$ is a multiplication lattice $L$-module.

Proof. Assume that $N$ is a weakly 2-absorbing element of $M$. Let $0 \neq a b c \leqslant(N$ : $\left.I_{M}\right)$ such that $a b \nless\left(N: I_{M}\right)$ and $b c \nless\left(N: I_{M}\right)$ for $a, b, c \in L$. If $a c b I_{M}=O_{M}$ then as $M$ is faithful we have $a b c \leqslant\left(O_{M}: I_{M}\right)=0$; a contradiction. Now as $N$ is a weakly 2-absorbing element with $O_{M} \neq a c\left(b I_{M}\right) \leqslant N, a\left(b I_{M}\right) \nless N$ and $c\left(b I_{M}\right) \nless N$ we have $a c \leqslant\left(N: I_{M}\right)$ which implies $\left(N: I_{M}\right)$ is a 2-absorbing element of $L$. Conversely assume that $\left(N: I_{M}\right)$ is a weakly 2-absorbing element of 
$L$ and $M$ is a multiplication lattice $L$-module. Let $O_{M} \neq a b Q \leqslant N$ for $a, b \in L$, $Q \in M$. Since $M$ is a multiplication lattice $L$-module, $Q=q I_{M}$ for some $q \in L$. If $a b q=0$, then $a b Q=O_{M}$, a contradiction. Now as $0 \neq a b q \leqslant\left(N: I_{M}\right)$ and since $\left(N: I_{M}\right)$ is a weakly 2 -absorbing element we have either $a b \leqslant\left(N: I_{M}\right)$ or $b q \leqslant\left(N: I_{M}\right)$ or $a q \leqslant\left(N: I_{M}\right)$ which implies either $a b \leqslant\left(N: I_{M}\right)$ or $b Q \leqslant N$ or $a Q \leqslant N$ and hence $N$ is a 2-absorbing element of $M$.

Result similar to Theorem 2.12 for a weakly 2-absorbing element of $M$ is as follows.

Theorem 2.15. Let $M$ be a faithful multiplication PG-lattice L-module with $I_{M}$ compact where $L$ is also PG-lattice. Then the following statements are equivalent:

(1) $N$ is a weakly 2-absorbing element of $M$.

(2) $\left(N: I_{M}\right)$ is a weakly 2-absorbing element of $L$.

(3) $N=q I_{M}$ for some weakly 2-absorbing element $q \in L$.

Proof. The proof is similar to the proof of Theorem 2.12 and hence omitted.

Thus a proper element $N$ of a multiplication lattice $L$-module $M$ is a 2-absorbing element if and only if $\left(N: I_{M}\right)$ is a 2-absorbing element of $L$ and a proper element $N$ of a faithful multiplication lattice $L$-module $M$ is a weakly 2-absorbing element if and only if $\left(N: I_{M}\right)$ is a weakly 2-absorbing element of $L$.

Theorem 2.16. If a proper element $N$ of an $L$-module $M$ is prime, then $N$ is a $(2,1)$-absorbing element. The converse holds if $M$ is a multiplication lattice $L$ module.

Proof. Assume that $N \in M$ is prime. Let $a b Q \leqslant N$ for $a, b \in L, Q \in M$. Then as $N$ is prime we have either $a \leqslant\left(N: I_{M}\right)$ or $b \leqslant\left(N: I_{M}\right)$ or $Q \leqslant N$ and we are done. Conversely assume that $N \in M$ is $(2,1)$-absorbing. Let $a Q \leqslant N$ for $a \in L$, $Q \in M$. Since $M$ is a multiplication lattice $L$-module, $Q=q I_{M}$ for some $q \in L$. Then as $a\left(q I_{M}\right) \leqslant N$ and $N$ is $(2,1)$-absorbing we have either $a \leqslant\left(N: I_{M}\right)$ or $q \leqslant\left(N: I_{M}\right)$ which implies either $a \leqslant\left(N: I_{M}\right)$ or $Q=q I_{M} \leqslant N$ and hence $N$ is prime.

Theorem 2.17. If a proper element $N$ of an L-module $M$ is weakly prime, then $N$ is a weakly $(2,1)$-absorbing element. The converse holds if $M$ is a multiplication lattice $L$-module.

Proof. The proof is similar to the proof of Theorem 2.16 and hence omitted. 
Theorem 2.18. If a proper element $N$ of an L-module $M$ is 2-absorbing, then $N$ is a $(3,2)$-absorbing element. The converse holds if $M$ is a multiplication lattice L-module.

Proof. Assume that $N \in M$ is 2-absorbing. Let $a b c Q \leqslant N$ for $a, b, c \in L, Q \in M$. Then by repeated use of the fact that $N$ is 2-absorbing we get either $a b \leqslant\left(N: I_{M}\right)$ or $[a(c Q) \leqslant N]$ or $[b(c Q) \leqslant N]$ which implies either $a b \leqslant\left(N: I_{M}\right)$ or $\left[a c \leqslant\left(N: I_{M}\right)\right.$ or $a Q \leqslant N$ or $c Q \leqslant N]$ or $\left[b c \leqslant\left(N: I_{M}\right)\right.$ or $\left.b Q \leqslant N\right]$. It follows that $N$ is $(3,2)$-absorbing. Conversely assume that $N$ is a $(3,2)$-absorbing element of a multiplication lattice $L$-module $M$. Let $a b Q \leqslant N$ for $a, b \in L, Q \in M$. Since $M$ is a multiplication lattice $L$-module, $Q=q I_{M}$ for some $q \in L$. Then as $a b\left(q I_{M}\right) \leqslant N$ and $N$ is $(3,2)$-absorbing we have either $\left[a b I_{M} \leqslant N\right.$ or $b q I_{M} \leqslant N$ or $\left.a q I_{M} \leqslant N\right]$ or $\left[a I_{M} \leqslant N\right.$ or $b I_{M} \leqslant N$ or $\left.q I_{M} \leqslant N\right]$ which implies either $\left[a b I_{M} \leqslant N\right.$ or $b q I_{M} \leqslant N$ or $\left.a q I_{M} \leqslant N\right]$ or $\left[b a I_{M} \leqslant N\right.$ or $q b I_{M} \leqslant N$ or $\left.a q I_{M} \leqslant N\right]$. It follows that either $a b \leqslant\left(N: I_{M}\right)$ or $b Q \leqslant N$ or $a Q \leqslant N$ and hence $N$ is 2-absorbing.

Theorem 2.19. If a proper element $N$ of an L-module $M$ is weakly 2-absorbing, then $N$ is a weakly $(3,2)$-absorbing element. The converse holds if $M$ is a multiplication lattice L-module.

Proof. The proof is similar to the proof of Theorem 2.18 and hence omitted.

Theorem 2.20. Let $N$ be a proper element of an $L$-module $M$ and $n, k \in \mathbb{Z}_{+}$such that $n>k$.

(1) If $N$ is $(n, k)$-absorbing, then $N$ is $(k+1, k)$-absorbing.

(2) If $N$ is $(n, k)$-absorbing, then $N$ is $\left(n, k^{\prime}\right)$-absorbing for every positive integer $k^{\prime}>k$.

Proof. (1) Assume that $N \in M$ is $(n, k)$-absorbing. Let $a_{1} a_{2} \cdots a_{n} Q \leqslant N$ where $a_{1}, a_{2}, \cdots, a_{n} \in L, Q \in M$. Since $N$ is $(n, k)$-absorbing it follows that either the product of any $k$ of the $a_{i}^{\prime} s$ is less than or equal to $\left(N: I_{M}\right)$ or there are $(k-1)$ of the $a_{i}^{\prime} s$ whose product with $Q$ is less than or equal to $N$ and hence $N$ is $(k+1, k)$ absorbing.

(2) Assume that $N \in M$ is $(n, k)$-absorbing. Let $k^{\prime} \in \mathbb{Z}_{+}$such that $k^{\prime}>k$. Let $a_{1} a_{2} \cdots a_{n} Q \leqslant N$ where $a_{1}, a_{2}, \cdots, a_{n} \in L, Q \in M$. Since $N$ is $(n, k)$-absorbing, we have either $b_{1} b_{2} \cdots b_{k} \leqslant\left(N: I_{M}\right)$ or $c_{1} c_{2} \cdots c_{k-1} Q \leqslant N$ where these $b_{i}^{\prime} s$ and $c_{i}^{\prime} s$ are some of the $a_{i}^{\prime} s$ obtained on renaming. It follows that either $b b_{1} b_{2} \cdots b_{k} \leqslant\left(N: I_{M}\right)$ for any element $b$ among $a_{i}^{\prime} s$ but other than $b_{i}^{\prime} s$ or $c c_{1} c_{2} \cdots c_{k-1} Q \leqslant N$ for any element $c$ among $a_{i}^{\prime} s$ but other than $c_{i}^{\prime} s$ and hence continuing the same argument we get $N$ is $\left(n, k^{\prime}\right)$-absorbing. 
Theorem 2.21. Let $N$ be a proper element of an $L$-module $M$ and $n, k \in \mathbb{Z}_{+}$such that $n>k$.

(1) If $N$ is weakly $(n, k)$-absorbing, then $N$ is weakly $(k+1, k)$-absorbing.

(2) If $N$ is weakly $(n, k)$-absorbing, then $N$ is weakly $\left(n, k^{\prime}\right)$-absorbing for every positive integer $k^{\prime}>k$.

Proof. The proof is similar to the proof of Theorem 2.20 and hence omitted.

Corollary 2.13 for an $n$-absorbing element of an $L$-module $M$ is as follows.

Theorem 2.22. Let $n \in \mathbb{Z}_{+}$. If a proper element $N$ of an L-module $M$ is $n$ absorbing, then $\left(N: I_{M}\right)$ is an n-absorbing element of L. The converse holds if $M$ is a multiplication lattice L-module.

Proof. Let $N$ be an $n$-absorbing element of $M$ and let $\widehat{a}_{i}=a_{1} \cdots a_{i-1} a_{i+1} \cdots a_{n}$ where $i(1 \leqslant i \leqslant n)$ and $a_{1}, \cdots, a_{n} \in L$. Assume that $a_{1} \cdots a_{n} a_{n+1} \leqslant\left(N: I_{M}\right)$ and $\widehat{a}_{i} a_{n+1} \nless\left(N: I_{M}\right)$ for every $i(1 \leqslant i \leqslant n)$. Then as $N$ is $n$-absorbing, $a_{1} \cdots a_{n}\left(a_{n+1} I_{M}\right) \leqslant N$ and $\widehat{a}_{i} a_{n+1} I_{M} \nless N$ we have $a_{1} \cdots a_{n} \leqslant\left(N: I_{M}\right)$ which implies $\left(N: I_{M}\right)$ is an $n$-absorbing element of $L$. Conversely assume that $\left(N: I_{M}\right)$ is an $n$-absorbing element of $L$ and $M$ is a multiplication lattice $L$-module. Let $a_{1} \cdots a_{n} Q \leqslant N$ for $a_{1}, \cdots, a_{n} \in L, Q \in M$. Since $M$ is a multiplication lattice $L$-module, $Q=q I_{M}$ for some $q \in L$. Then as $a_{1} \cdots a_{n} q \leqslant\left(N: I_{M}\right)$ and since $\left(N: I_{M}\right)$ is an $n$-absorbing element we have either $a_{1} \cdots a_{n} \leqslant\left(N: I_{M}\right)$ or there exist $(n-1)$ of $a_{i}^{\prime} s$ whose product with $q$ is less than or equal to $\left(N: I_{M}\right)$ which implies either $a_{1} \cdots a_{n} \leqslant\left(N: I_{M}\right)$ or there exist $(n-1)$ of $a_{i}^{\prime} s$ whose product with $q I_{M}=Q$ is less than or equal to $N$ and hence $N$ is an $n$-absorbing element of $M$.

Lemma 2.23. Let $m, n \in \mathbb{Z}_{+}$. If a proper element $N$ of an L-module $M$ is $n$ absorbing then $N$ is an m-absorbing element of $M$ for all $m>n$.

Proof. Let $m, n \in \mathbb{Z}_{+}$be such that $m>n$. Let $x_{1} \cdots x_{m} Q=x_{1} \cdots x_{n}\left(x_{n+1} \cdots x_{m} Q\right) \leqslant$ $N$ for $x_{1}, \cdots, x_{m} \in L, Q \in M$. Then as $N$ is $n$-absorbing, we have either $x_{1} \cdots x_{n} \leqslant$ $\left(N: I_{M}\right)$ or $x_{1} \cdots x_{i-1} x_{i+1} \cdots x_{n}\left(x_{n+1} \cdots x_{m} Q\right) \leqslant N$ for some $i(1 \leqslant i \leqslant n)$ which implies either $x_{1} \cdots x_{n} \cdots x_{m} \leqslant\left(N: I_{M}\right)$ or $\left(x_{1} \cdots x_{i-1} x_{i+1} \cdots x_{n} x_{n+1} \cdots x_{m}\right) Q \leqslant N$ for some $i(1 \leqslant i \leqslant m)$ and thus $N$ is an $m$-absorbing element of $M$.

In view of above Lemma 2.23 , we have the following definition.

Definition 2.24. If a proper element $N$ is an $n$-absorbing element of $M$ for some $n \in \mathbb{Z}_{+}$, then we define $\omega(N)=\min \left\{n \in \mathbb{Z}_{+} \mid N\right.$ is an $n$-absorbing element of $\left.M\right\}$ otherwise we write $\omega(N)=\infty$. Moreover we define $\omega\left(I_{M}\right)=0$. 
Thus for any element $N \in M$ we have $\omega(N) \in \mathbb{Z}_{+} \cup\{0, \infty\}$ with $\omega(N)=1$ if and only if $N$ is a prime element of $M$ and $\omega(N)=0$ if and only if $N=I_{M}$. So $\omega(N)$ measures in some sense how far ' $N^{\prime}$ is from being a prime element of $M$.

Theorem 2.25. If a proper element $N$ of an $L$-module $M$ is p-primary such that $p^{n} I_{M} \leqslant N$ where $n \in \mathbb{Z}_{+}$, then $N$ is an n-absorbing element of $M$. Moreover, $\omega(N) \leqslant n$.

Proof. Let $a_{1} \cdots a_{n} Q \leqslant N$ with $\widehat{a}_{i} Q \nless N$ for every $i(1 \leqslant i \leqslant n)$ where $\widehat{a}_{i}$ is the element $a_{1} \cdots a_{i-1} a_{i+1} \cdots a_{n}$ and $a_{1}, \cdots, a_{n} \in L, Q \in M$. As $N$ is $p$-primary, $a_{i}\left(\widehat{a}_{i} Q\right) \leqslant N$ and $\widehat{a}_{i} Q \nless N$, we have $a_{i} \leqslant \sqrt{N: I_{M}}=p$ for every $i(1 \leqslant i \leqslant n)$ which implies $a_{1} \cdots a_{n} \leqslant p^{n}$. It follows that $a_{1} \cdots a_{n} \leqslant\left(N: I_{M}\right)$ and thus $N$ is an $n$-absorbing element of $M$. The "moreover" statement is clear.

Corollary 2.26. Let a proper element $N$ of an L-module $M$ be p-primary. Then $N$ is 2-absorbing if and only if $p^{2} I_{M} \leqslant N$.

Proof. Let a $p$-primary element $N \in M$ be 2 -absorbing. Then by Corollary 2.13 $\left(N: I_{M}\right)$ is a 2 -absorbing element of $L$ which implies $\left(\sqrt{N: I_{M}}\right)^{2} \leqslant\left(N: I_{M}\right)$ by Lemma 2(iii) of [11] and thus $p^{2} I_{M} \leqslant N$. The converse part is clear by Theorem 2.25 .

We define a classical prime element of an $L$-module $M$ as follows.

Definition 2.27. A proper element $N \in M$ is said to be classical prime if for each element $K \in M$ and elements $a, b \in L ; a b K \leqslant N$ implies either $a K \leqslant N$ or $b K \leqslant N$.

Theorem 2.28. Let $N$ be a proper element of an L-module $M$. Then $N$ is prime implies $N$ is classical prime implies $N$ is 2-absorbing implies $N$ is weakly 2-absorbing.

Proof. Assume that $N \in M$ is prime. Let $a b K \leqslant N$ for $a, b \in L, K \in M$. Then as $N$ is prime we have either $a \leqslant\left(N: I_{M}\right) \leqslant(N: K)$ or $b K \leqslant N$ which implies either $a K \leqslant N$ or $b K \leqslant N$ and thus $N$ is classical prime. Now let $N$ be classical prime and let $a b K \leqslant N$ for $a, b \in L, K \in M$. Then as $N$ is classical prime we have either $a K \leqslant N$ or $b K \leqslant N$ and thus $N$ is 2-absorbing. Last implication is obvious since every 2 -absorbing element is weakly 2 -absorbing.

From the above Theorem 2.28, it is clear that every 2-absorbing element is weakly 2-absorbing. But the converse is not true as shown in the following example. 
Example 2.29. Let $R=\mathbb{Z}$ and $M=\mathbb{Z} /(30 \mathbb{Z})$. Then $M$ is a module over $\mathbb{Z}$. Suppose that $L(R)$ is the set of all ideals of $R$ and $L(M)$ is the set of all submodules of $M$. Then $L(M)$ is a lattice module over $L(R)$. Obviously $N=\{30 \mathbb{Z}\}$ being the zero element of $L(M)$ is weakly 2-absorbing. However $N$ is not a 2-absorbing element of $L(M)$ since $(2)(3)(5+30 \mathbb{Z}) \subseteq N$ and $(2)(3) \nsubseteq(N: M),(2)(5+30 \mathbb{Z}) \nsubseteq$ $N,(3)(5+30 \mathbb{Z}) \nsubseteq N$.

The following theorem shows that under particular condition a weakly 2-absorbing element of an $L$-module $M$ is 2 -absorbing.

Theorem 2.30. If a weakly 2-absorbing element $N$ of an $L$-module $M$ is such that $\left(N: I_{M}\right)^{2} N \neq O_{M}$, then $N$ is a 2-absorbing element.

Proof. Assume that $\left(N: I_{M}\right)^{2} N \neq O_{M}$. Let $a b Q \leqslant N$ for $a, b \in L, Q \in M$. If $a b Q \neq O_{M}$, then as $N$ is weakly 2 -absorbing we get either $a b \leqslant\left(N: I_{M}\right)$ or $a Q \leqslant N$ or $b Q \leqslant N$ and we are done. So let $a b Q=O_{M}$. First assume that $a b N \neq O_{M}$. Then $a b N_{0} \neq O_{M}$ for some $N_{0} \leqslant N$ in $M$. As $O_{M} \neq a b\left(Q \vee N_{0}\right) \leqslant N$ and $N$ is weakly 2 -absorbing we have either $a b \leqslant\left(N: I_{M}\right)$ or $a\left(Q \vee N_{0}\right) \leqslant N$ or $b\left(Q \vee N_{0}\right) \leqslant N$ which implies either $a b \leqslant\left(N: I_{M}\right)$ or $a Q \leqslant N$ or $b Q \leqslant N$ and we are done. Hence we may assume that $a b N=O_{M}$. If $a\left(N: I_{M}\right) Q \neq O_{M}$, then $a r_{0} Q \neq O_{M}$ for some $r_{0} \leqslant\left(N: I_{M}\right)$ in $L$. Since $O_{M} \neq a r_{0} Q \leqslant a\left(b \vee r_{0}\right) Q \leqslant N$ and $N$ is weakly 2-absorbing we have either $a\left(b \vee r_{0}\right) \leqslant\left(N: I_{M}\right)$ or $a Q \leqslant N$ or $\left(b \vee r_{0}\right) Q \leqslant N$ which implies either $a b \leqslant\left(N: I_{M}\right)$ or $a Q \leqslant N$ or $b Q \leqslant N$ and we are done. So we can assume that $a\left(N: I_{M}\right) Q=O_{M}$. Likewise we can assume that $b\left(N: I_{M}\right) Q=O_{M}$. As $\left(N: I_{M}\right)^{2} N \neq O_{M}$, there exist $a_{0}, b_{0} \leqslant\left(N: I_{M}\right)$ and $X_{0} \leqslant N$ with $a_{0} b_{0} X_{0} \neq$ $O_{M}$. If $a b_{0} X_{0} \neq O_{M}$ then $O_{M} \neq a b_{0} X_{0} \leqslant a\left(b \vee b_{0}\right)\left(Q \vee X_{0}\right) \leqslant N$. As $N$ is weakly 2absorbing we get either $a\left(b \vee b_{0}\right) \leqslant\left(N: I_{M}\right)$ or $a\left(Q \vee X_{0}\right) \leqslant N$ or $\left(b \vee b_{0}\right)\left(Q \vee X_{0}\right) \leqslant$ $N$ which implies either $a b \leqslant\left(N: I_{M}\right)$ or $a Q \leqslant N$ or $b Q \leqslant N$ and we are done. So we can assume that $a b_{0} X_{0}=O_{M}$. Likewise we can assume that $a_{0} b_{0} Q=O_{M}$ and $a_{0} b X_{0}=O_{M}$. Then as $O_{M} \neq a_{0} b_{0} X_{0} \leqslant\left(a \vee a_{0}\right)\left(b \vee b_{0}\right)\left(Q \vee X_{0}\right) \leqslant N$ and $N$ is weakly 2-absorbing we get either $\left(a \vee a_{0}\right)\left(b \vee b_{0}\right) \leqslant\left(N: I_{M}\right)$ or $\left(a \vee a_{0}\right)\left(Q \vee X_{0}\right) \leqslant N$ or $\left(b \vee b_{0}\right)\left(Q \vee X_{0}\right) \leqslant N$ which implies either $a b \leqslant\left(N: I_{M}\right)$ or $a Q \leqslant N$ or $b Q \leqslant N$ and thus $N$ is a 2-absorbing element.

We define a nilpotent element of an $L$-module $M$ in the following manner.

Definition 2.31. A proper element $N$ of an $L$-module $M$ is said to be nilpotent if $\left(N: I_{M}\right)^{k} N=O_{M}$ for some $k \in \mathbb{Z}_{+}$.

The consequences of Theorem 2.30 are presented in the form of following corollaries. 
Corollary 2.32. If a proper element $N$ of an L-module $M$ is weakly 2-absorbing but not 2-absorbing, then $N$ is a nilpotent element of $M$.

Proof. The proof is obvious.

Corollary 2.33. If a proper element $N$ of an L-module $M$ is weakly 2-absorbing but not 2-absorbing, then $\left(N: I_{M}\right)^{3} N=O_{M}$.

Proof. As $\left(N: I_{M}\right)^{3} \leqslant\left(N: I_{M}\right)^{2}$, we have $\left(N: I_{M}\right)^{3} N \leqslant\left(N: I_{M}\right)^{2} N=O_{M}$ by Theorem 2.30 and hence $\left(N: I_{M}\right)^{3} N=O_{M}$.

Corollary 2.34. If a proper element $N$ of an L-module $M$ is weakly 2-absorbing but not 2-absorbing, then $\left(N: I_{M}\right)^{n} N=O_{M}$ for every $n \geqslant 3$.

Proof. The proof is obvious.

Corollary 2.35. If a proper element $N$ of a multiplication lattice $L$-module $M$ is weakly 2-absorbing but not 2-absorbing, then $\left(N: I_{M}\right)^{3} I_{M}=O_{M}$.

Proof. Since $M$ is a multiplication lattice $L$-module, we have $N=\left(N: I_{M}\right) I_{M}$. By Theorem 2.30, we have $\left(N: I_{M}\right)^{2} N=O_{M}$ which implies $\left(N: I_{M}\right)^{3} I_{M}=O_{M}$.

Corollary 2.36. If a proper element $N$ of a faithful multiplication lattice L-module $M$ is weakly 2-absorbing but not 2-absorbing, then $\left(N: I_{M}\right) \leqslant \sqrt{0}$ and hence $\sqrt{N: I_{M}}=\sqrt{0}$. Moreover, if $L$ is a reduced lattice then $\left(N: I_{M}\right)=0$.

Proof. The proof is obvious.

Corollary 2.37. Let $L$ be a reduced lattice. If $O_{M}<N<I_{M}$ is a weakly 2absorbing element of a faithful multiplication lattice L-module $M$, then $N$ is a 2-absorbing element of $M$.

Proof. The proof is obvious.

\section{3. $\operatorname{rad}(N)$ as a 2-absorbing element of $M$}

In this section, we prove $\operatorname{rad}(N)$ is a 2 -absorbing element of an $L$-module $M$ if $N \in M$ is a 2 -absorbing element. We begin with defining the radical of an element of a lattice module. In view of the definition of the $M$-radical of a submodule of an $R$-module $M$ in [12], the definition of the radical of an element of an $L$-module $M$ is as follows.

Definition 3.1. Let $N$ be a proper element of an $L$-module $M$. Then the radical of $N$ is denoted as $\operatorname{rad}(N)$ and is defined as the element $\wedge\{P \in M \mid P$ is a prime element and $N \leqslant P\}$. If $N \nless P$ for any prime $P \in M$, then we write $\operatorname{rad}(N)=I_{M}$. 
Before proving $\operatorname{rad}(N)$ is a 2-absorbing element of $M$, we prove the results required to show that $\operatorname{rad}\left(a I_{M}\right)=\sqrt{a} I_{M}$ as proved in an $R$-module $M$ in [10].

Lemma 3.2. Let $L$ be a $P G$-lattice and $M$ be a faithful multiplication PG-lattice L-module. Then $\underset{\alpha \in \triangle}{\wedge}\left(a_{\alpha} I_{M}\right)=\left(\underset{\alpha \in \triangle}{\wedge} a_{\alpha}\right) I_{M}$ where $\left\{a_{\alpha} \in L \mid \alpha \in \triangle\right\}$.

Proof. Clearly $\left(\underset{\alpha \in \triangle}{\wedge} a_{\alpha}\right) I_{M} \leqslant \wedge_{\alpha \in \triangle}\left(a_{\alpha} I_{M}\right)$. Let $X \leqslant \bigwedge_{\alpha \in \triangle}\left(a_{\alpha} I_{M}\right)$ where $X \in M$. We may suppose that $X$ is a principal element. Assume that $\left(\left(\underset{\alpha \in \triangle}{\wedge} a_{\alpha}\right) I_{M}: X\right) \neq 1$. Then there exists a maximal element $q \in L$ such that $\left(\left(\wedge_{\alpha \in \triangle} a_{\alpha}\right) I_{M}: X\right) \leqslant q$. As $M$ is a multiplication lattice $L$-module and $q \in L$ is maximal, by Theorem 4 of [7], two cases arise:

Case 1. For principal element $X \in M$, there exists a principal element $r \in L$ with $r \notin q$ such that $r X=O_{M}$. Then $r \leqslant\left(O_{M}: X\right) \leqslant\left(\left(\wedge_{\alpha \in \triangle} a_{\alpha}\right) I_{M}: X\right) \leqslant q$ which is a contradiction.

Case 2. There exists a principal element $Y \in M$ and a principal element $b \in L$ with $b \notin q$ such that $b I_{M} \leqslant Y$. Then $b X \leqslant Y, b X \leqslant b\left[\wedge_{\alpha \in \triangle}\left(a_{\alpha} I_{M}\right)\right] \leqslant$ $\bigwedge_{\alpha \in \triangle}\left(a_{\alpha} b I_{M}\right) \leqslant \bigwedge_{\alpha \in \triangle}\left(a_{\alpha} Y\right)$ and $\left(O_{M}: Y\right) b I_{M} \leqslant\left(O_{M}: Y\right) Y=O_{M}$ since $Y$ is meet principal. As $M$ is faithful it follows that $b\left(O_{M}: Y\right)=0$. Since $Y$ is meet principal, $(b X: Y) Y=b X \wedge Y=b X$. Let $s=(b X: Y)$ then $s Y=b X \leqslant \wedge_{\alpha \in \triangle}\left(a_{\alpha} Y\right)$. So $s=(b X: Y)=(s Y: Y) \leqslant\left[\wedge_{\alpha \in \triangle}\left(a_{\alpha} Y\right): Y\right]=\wedge_{\alpha \in \triangle}\left(a_{\alpha} Y: Y\right)=\wedge_{\alpha \in \triangle}\left[a_{\alpha} \vee\left(O_{M}: Y\right)\right]$ since $Y$ is join principal. Therefore $b s \leqslant b\left[\bigwedge_{\alpha \in \triangle}\left[a_{\alpha} \vee\left(O_{M}: Y\right)\right]\right] \leqslant \wedge_{\alpha \in \triangle}\left[b\left[a_{\alpha} \vee\left(O_{M}\right.\right.\right.$ : $Y)]]=\wedge_{\alpha \in \triangle}\left[\left(b a_{\alpha}\right) \vee b\left(O_{M}: Y\right)\right]=\wedge_{\alpha \in \triangle}\left(b a_{\alpha}\right) \leqslant b \wedge\left(\underset{\alpha \in \triangle}{\wedge} a_{\alpha}\right) \leqslant\left(\underset{\alpha \in \triangle}{\wedge} a_{\alpha}\right)$ and so $b^{2} X=b(b X)=b s Y \leqslant\left(\wedge_{\alpha \in \triangle} a_{\alpha}\right) Y \leqslant\left(\wedge_{\alpha \in \triangle} a_{\alpha}\right) I_{M}$. Hence $b^{2} \leqslant\left(\left(\bigwedge_{\alpha \in \triangle} a_{\alpha}\right) I_{M}: X\right) \leqslant q$ which implies $b \leqslant \sqrt{q}=q$; a contradiction.

Thus the assumption that $\left(\left(\wedge_{\alpha \in \triangle} a_{\alpha}\right) I_{M}: X\right) \neq 1$ is absurd and so we must have $\left(\left(\wedge_{\alpha \in \triangle} a_{\alpha}\right) I_{M}: X\right)=1$ which implies $X \leqslant\left(\wedge_{\alpha \in \triangle}^{\wedge} a_{\alpha}\right) I_{M}$. It follows that $\hat{\alpha \in \triangle}_{\alpha \in}\left(a_{\alpha} I_{M}\right) \leqslant$ $\left(\wedge_{\alpha \in \triangle}^{\wedge} a_{\alpha}\right) I_{M}$ and hence $\underset{\alpha \in \triangle}{\wedge}\left(a_{\alpha} I_{M}\right)=\left(\wedge_{\alpha \in \triangle}^{\wedge} a_{\alpha}\right) I_{M}$.

Lemma 3.3. Let $L$ be a $P G$-lattice and $M$ be a faithful multiplication PG-lattice $L$-module with $I_{M}$ compact. If a proper element $q \in L$ is a prime element, then $q I_{M}$ is a prime element of $M$.

Proof. As $I_{M}$ is compact and $q \in L$ is proper by Theorem 5 of [7] we have $q I_{M} \neq$ $I_{M}$. Let $a X \leqslant q I_{M}$ and $a \nless\left(q I_{M}: I_{M}\right)$ for $a \in L, X \in M$. Then $a \not z q$. We may suppose that $X$ is a principal element. Assume that $\left(\left(q I_{M}\right): X\right) \neq 1$. Then there exists a maximal element $m \in L$ such that $\left(\left(q I_{M}\right): X\right) \leqslant m$. As $M$ is a multiplication lattice $L$-module and $m \in L$ is maximal, by Theorem 4 of [7], two cases arise: 
Case 1. For principal element $X \in M$, there exists a principal element $r \in L$ with $r \notin m$ such that $r X=O_{M}$. Then $r \leqslant\left(O_{M}: X\right) \leqslant\left(\left(q I_{M}\right): X\right) \leqslant m$ which is a contradiction.

Case 2. There exists a principal element $Y \in M$ and a principal element $b \in L$ with $b \nless m$ such that $b I_{M} \leqslant Y$. Then $b X \leqslant Y, b a X \leqslant b q I_{M}=q\left(b I_{M}\right) \leqslant q Y$ and $\left(O_{M}: Y\right) b I_{M} \leqslant\left(O_{M}: Y\right) Y=O_{M}$ since $Y$ is meet principal. As $M$ is faithful it follows that $b\left(O_{M}: Y\right)=0$. Since $Y$ is meet principal, $(b X: Y) Y=b X$. Let $s=(b X: Y)$ then $s Y=b X$ and so $a s Y=a b X \leqslant q Y$. Since $Y$ is meet principal, $a b X=(a b X: Y) Y=c Y$ where $c=(a b X: Y)$. Since $c Y=a b X \leqslant q Y$ and $Y$ is join principal we have $c \vee\left(O_{M}: Y\right)=(c Y: Y) \leqslant(q Y: Y)=q \vee\left(O_{M}: Y\right)$. So $b c \leqslant b q \leqslant q$. On the other hand since $Y$ is join principal, $c=(a b X: Y)=(a s Y$ : $Y)=a s \vee\left(O_{M}: Y\right)$ and so $a b s \leqslant a b s \vee b\left(O_{M}: Y\right)=b\left(a s \vee\left(O_{M}: Y\right)\right)=b c \leqslant q$. If $b \leqslant q$, then $b \leqslant q \leqslant\left(\left(q I_{M}\right): X\right) \leqslant m$ which contradicts $b \nless m$ and so $b \nless q$. Now as $a b s \leqslant q, a \nless q, b \nless q$ and $q$ is prime, we have $s \leqslant q$. Hence $b X=s Y \leqslant q Y \leqslant\left(q I_{M}\right)$ which implies $b \leqslant\left(\left(q I_{M}\right): X\right) \leqslant m$; a contradiction.

Thus the assumption that $\left(\left(q I_{M}\right): X\right) \neq 1$ is absurd and so we must have $\left(\left(q I_{M}\right): X\right)=1$ which implies $X \leqslant\left(q I_{M}\right)$. Therefore $q I_{M}$ is a prime element of $M$.

Lemma 3.4. In an $L$-module $M$, if a proper element $Q \in M$ is prime such that $X \leqslant Q$, then $\left(Q: I_{M}\right) \in L$ is prime such that $\sqrt{X: I_{M}} \leqslant\left(Q: I_{M}\right)$ where $X \in M$ is a proper element.

Proof. Obviously, $\left(Q: I_{M}\right) \in L$ is prime by Proposition 3.6 of [1]. Further, if $a \leqslant \sqrt{X: I_{M}}$, then $a^{n} \leqslant\left(X: I_{M}\right) \leqslant\left(Q: I_{M}\right)$ for some $n \in \mathbb{Z}_{+}$which implies $a \leqslant\left(Q: I_{M}\right)$ and so $\sqrt{X: I_{M}} \leqslant\left(Q: I_{M}\right)$.

Lemma 3.5. For every proper element $N$ of an L-module $M,\left(\sqrt{N: I_{M}}\right) I_{M} \leqslant$ $\operatorname{rad}(N)$.

Proof. Let $P \in M$ be prime such that $N \leqslant P$. Then by Lemma 3.4, $\left(P: I_{M}\right) \in L$ is prime such that $\sqrt{N: I_{M}} \leqslant\left(P: I_{M}\right)$ which implies $\left(\sqrt{N: I_{M}}\right) I_{M} \leqslant P$. Thus whenever $P \in M$ is prime such that $N \leqslant P$ we have $\left(\sqrt{N: I_{M}}\right) I_{M} \leqslant P$. It follows that $\left(\sqrt{N: I_{M}}\right) I_{M} \leqslant \operatorname{rad}(N)$.

Theorem 3.6. Let $L$ be a PG-lattice and $M$ be a faithful multiplication PG-lattice $L$-module with $I_{M}$ compact. Then $\operatorname{rad}(N)=\sqrt{a} I_{M}$ for every proper element $N=$ $a I_{M}$ of $M$ where $a=\left(N: I_{M}\right) \in L$.

Proof. Let $b=\wedge\{p \in L \mid p$ is a prime element and $a \leqslant p\}=\sqrt{a}$. Then by

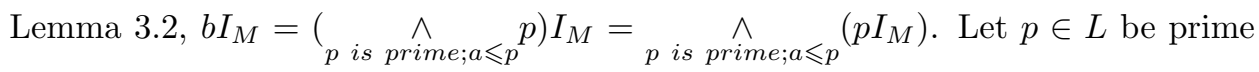


such that $a \leqslant p$. Also as $p \in L$ is a prime element by Lemma 3.3 we have $p I_{M} \in M$ is a prime element. Then $N=a I_{M} \leqslant p I_{M}$ and so $\operatorname{rad}(N) \leqslant p I_{M}$. It follows that $\operatorname{rad}(N) \leqslant \widehat{p}_{p \text { is prime; } a \leqslant p}\left(p I_{M}\right)=b I_{M}$ and hence $\operatorname{rad}(N) \leqslant \sqrt{a} I_{M}$. But by Lemma 3.5 we have $\sqrt{a} I_{M} \leqslant \operatorname{rad}(N)$. Therefore $\operatorname{rad}(N)=\sqrt{a} I_{M}$.

Following corollary is an outcome of Corollary 2.35 and Theorem 3.6.

Corollary 3.7. Let $L$ be a $P G$-lattice and $M$ be a faithful multiplication PG-lattice $L$-module with $I_{M}$ compact. If a proper element $N$ of $M$ is weakly 2-absorbing but not 2-absorbing, then $N \leqslant \operatorname{rad}\left(O_{M}\right)$.

Proof. As $O_{M}=\left(O_{M}: I_{M}\right) I_{M}=0 I_{M}$, we have $\operatorname{rad}\left(O_{M}\right)=\sqrt{0} I_{M}$ by Theorem 3.6. By Corollary 2.35, we have $\left(N: I_{M}\right)^{3} I_{M}=O_{M}$ which implies $\left(N: I_{M}\right)^{3} \leqslant$ $\left(O_{M}: I_{M}\right)=0$ and hence $\left(N: I_{M}\right) \leqslant \sqrt{0}$. It follows that $N=\left(N: I_{M}\right) I_{M} \leqslant$ $\sqrt{0} I_{M}=\operatorname{rad}\left(O_{M}\right)$.

Lemma 3.8. In a multiplication lattice $L$-module $M$, the meet of each pair of distinct prime elements of $M$ is a 2-absorbing element.

Proof. Let $N$ and $K$ be any two distinct prime elements of $M$. Let $a b Q \leqslant(N \wedge K)$ with $a Q \nless(N \wedge K)$ and $b Q \nless(N \wedge K)$ for $a, b \in L, Q \in M$. Since $M$ is a multiplication lattice $L$-module, $Q=q I_{M}$ for some $q \in L$. Clearly $a Q \nless K$ and $b Q \nless N$ lead us to a contradiction because $N$ is prime and $a(b Q) \leqslant(N \wedge K) \leqslant N$ gives $a I_{M} \leqslant N$ which implies $q a I_{M}=a Q \leqslant N$. Similarly $a Q \nless K$ and $b Q \nless K$ lead us to a contradiction. So assume that $a Q \nless N$ and $b Q \nless K$. Now $a(b Q) \leqslant$ $(N \wedge K) \leqslant K, b Q \nless K, K$ is prime gives $a \leqslant\left(K: I_{M}\right)$ and $b(a Q) \leqslant(N \wedge K) \leqslant N$, $a Q \nless N, N$ is prime gives $b \leqslant\left(N: I_{M}\right)$. Hence $a b \leqslant(a \wedge b) \leqslant\left[\left(K: I_{M}\right) \wedge(N\right.$ : $\left.\left.I_{M}\right)\right]=\left[(N \wedge K): I_{M}\right]$ which implies $(N \wedge K)$ is a 2-absorbing element of $M$.

Now we are in a position to prove $\operatorname{rad}(N)$ is a 2-absorbing element of $M$ which is the main aim of this section.

Theorem 3.9. Let $L$ be a $P G$-lattice and $M$ be a faithful multiplication $P G$-lattice $L$-module with $I_{M}$ compact. If a proper element $N \in M$ is a 2-absorbing element, then $\operatorname{rad}(N)$ is a 2-absorbing element of $M$.

Proof. By Corollary 2.13, $\left(N: I_{M}\right)$ is a 2-absorbing element of $L$. By Theorem 3 of [11], two cases arise:

Case 1. $\sqrt{N: I_{M}}=p$ is a prime element of $L$. Then by Lemma 3.3 and Theorem 3.6, we have $\operatorname{rad}(N)=\left(\sqrt{N: I_{M}}\right) I_{M}=p I_{M}$ is prime and hence $\operatorname{rad}(N)$ is a 2absorbing element of $M$. 
Case 2. $\sqrt{N: I_{M}}=p_{1} \wedge p_{2}$ where $p_{1}, p_{2}$ are the only distinct prime elements of $L$ that are minimal over $\left(N: I_{M}\right)$. Then by Lemma $3.3, p_{1} I_{M}$ and $p_{2} I_{M}$ are distinct prime elements of $M$ and are minimal over $N$. So by Theorem 3.6 and Lemma 3.2, we have $\operatorname{rad}(N)=\left(\sqrt{N: I_{M}}\right) I_{M}=\left(p_{1} \wedge p_{2}\right) I_{M}=p_{1} I_{M} \wedge p_{2} I_{M}$. Hence by Lemma $3.8, \operatorname{rad}(N)$ is a 2 -absorbing element of $M$.

Theorem 3.10. Let $L$ be a $P G$-lattice and $M$ be a faithful multiplication $P G$-lattice $L$-module with $I_{M}$ compact. If a proper element $N \in M$ is 2-absorbing, then one of the following statement holds true:

(1) $\operatorname{rad}(N)=p I_{M}$ is a prime element of $M$ such that $p^{2} I_{M} \leqslant N$.

(2) $\operatorname{rad}(N)=p_{1} I_{M} \wedge p_{2} I_{M}$ and $\left(p_{1} p_{2}\right) I_{M} \leqslant N$ where $p_{1} I_{M}$ and $p_{2} I_{M}$ are the only distinct prime elements of $M$ that are minimal over $N$.

Proof. By Corollary 2.13, $\left(N: I_{M}\right)$ is a 2-absorbing element of $L$. Then by Theorem 3 of [11], we have either $\sqrt{N: I_{M}}=p$ is a prime element of $L$ such that $p^{2} \leqslant\left(N: I_{M}\right)$ or $\sqrt{N: I_{M}}=p_{1} \wedge p_{2}$ and $p_{1} p_{2} \leqslant\left(N: I_{M}\right)$ where $p_{1}$ and $p_{2}$ are the only distinct prime elements of $L$ that are minimal over $\left(N: I_{M}\right)$. By Theorem 3.6, Lemma 3.3 and Lemma 3.2, it follows that either $\operatorname{rad}(N)=p I_{M}$ is a prime element of $M$ such that $p^{2} I_{M} \leqslant N$ or $\operatorname{rad}(N)=\left(p_{1} \wedge p_{2}\right) I_{M}=p_{1} I_{M} \wedge p_{2} I_{M}$ and $\left(p_{1} p_{2}\right) I_{M} \leqslant N$ where $p_{1} I_{M}$ and $p_{2} I_{M}$ are the only distinct prime elements of $M$ that are minimal over $N$.

Note that if $N$ is a 2-absorbing element of a faithful multiplication PG-lattice $L$-module $M$ with $I_{M}$ compact, then $\left(\sqrt{N: I_{M}}\right) \operatorname{rad}(N) \leqslant N \leqslant \operatorname{rad}(N)$.

Acknowledgment. The authors would like to thank the referee for the valuable suggestions and comments.

\section{References}

[1] E. A. Al-Khouja, Maximal elements and prime elements in lattice modules, Damascus University Journal for BASIC SCIENCES, 19(2) (2003), 9-21.

[2] F. Alarcón, D. D. Anderson and C. Jayaram, Some results on abstract commutative ideal theory, Period. Math. Hungar., 30(1) (1995), 1-26.

[3] D. F. Anderson and A. Badawi, On n-absorbing ideals of commutative rings, Comm. Algebra, 39(5) (2011), 1646-1672.

[4] A. Badawi, On 2-absorbing ideals of commutative rings, Bull. Austral. Math. Soc., 75(3) (2007), 417-429.

[5] A. Badawi and A. Y. Darani, On weakly 2-absorbing ideals of commutative rings, Houston J. Math., 39(2) (2013), 441-452. 
[6] S. Ballal and V. Kharat, On generalization of prime, weakly prime and almost prime elements in multiplicative lattices, Int. J. Algebra, 8(9) (2014), 439-449.

[7] F. Callialp and U. Tekir, Multiplication lattice modules, Iran. J. Sci. Technol. Trans. A Sci., 35(4) (2011), 309-313.

[8] A. Y. Darani and F. Soheilnia, 2-absorbing and weakly 2-absorbing submodules, Thai J. Math., 9(3) (2011), 577-584.

[9] A. Y. Darani and F. Soheilnia, On n-absorbing submodules, Math. Commun., $17(2)(2012), 547-557$.

[10] Z. A. El-Bast and P. F. Smith, Multiplication modules, Comm. Algebra, 16(4) (1988), 755-779.

[11] C. Jayaram, U. Tekir and E. Yetkin, 2-absorbing and weakly 2-absorbing elements in multiplicative lattices, Comm. Algebra, 42(6) (2014), 2338-2353.

[12] R. L. McCasland and M. E. Moore, On radicals of submodules of finitely generated modules, Canad. Math. Bull., 29(1) (1986), 37-39.

\section{S. Manjarekar}

Department of Mathematics

Shivaji University, Vidyanagar

Kolhapur-416004

India

e-mail: csmanjrekar@yahoo.co.in

\section{A. V. Bingi}

Department of Mathematics

St. Xavier's College-autonomous

5, Mahapalika Marg

Mumbai-400001

India

e-mail: ashok.bingi@xaviers.edu 